Internat. J. Math. \& Math. Sci.

Vol. 1 (1978) 93-96

\title{
A NOTE ON RIESZ ELEMENTS IN C*-ALGEBRAS
}

\author{
DAVID LEGG \\ Department of Mathematics \\ Indiana University-Purdue University \\ Fort Wayne, Indiana \\ (Received December 5, 1977)
}

ABSTRACT. It is known that every Riesz operator $\mathrm{R}$ on a Hilbert space can be written $R=Q+C$, where $C$ is compact and both $Q$ and $C Q-Q C$ are quasinilpotent. This result is extended to a general $C^{*}$-algebra setting.

1. INTRODUCTION.

In [3], Smyth develops a Riesz theory for elements in a Banach algebra with respect to an ideal of algebraic elements. In [1], Chui, Smith and Ward show that every Riesz operator on a Hilbert space is decomposible into $R=Q+C$, where $C$ is compact and both $Q$ and $C Q-Q C$ are quasinilpotent. In this paper we use Smyth's work to show that the analogous result holds in an arbitrary $C^{*}$-algebra.

2. DEFINITIONS AND NOTATION.

Let $A$ be a $C^{*}$-algebra, and let $F$ be a two-sided ideal of algebralc elements 
of A. An element $T \varepsilon A$ is a Riesz element if its coset $T+\bar{F}$ in $A / \bar{F}$ has spectral radius 0 . A point $\lambda \varepsilon \sigma(T)$ is a finite pole of $T$ if it is isolated in $\sigma(T)$ and the corresponding spectral projection lies in $F$. Let $E \sigma(T)=\{\lambda \varepsilon \sigma(T): \lambda$ is not a finite pole of $T\}$. Smyth has shown that $T$ is a Riesz element if and only if $E \sigma(T) \subseteq\{0\},[3, T h m .5 .3]$. Smyth also showed that if $T$ is a Riesz element, then $T=Q+U$, where $Q$ is quasinilpotent and $U \varepsilon \bar{F}$. [3, Thm. 6.9]. This is a generalization of West's result [4, Thm. 7.5]. We now extend the result of Chui, Smith and Ward [1, Thm. 1] by showing that UQ - QU is quasinilpotent, where $T=Q+U$ is the Smyth decomposition.

3. OUTLINE OF SMYTH'S CONSTRUCTION.

Let $T$ be a Riesz element, and label the elements of $\sigma(T) \backslash E \sigma(T)$ by $\lambda_{n}, n=1,2, \ldots$, in such a way that $\left|\lambda_{n}\right| \geq\left|\lambda_{n+1}\right|, \lambda_{n} \rightarrow 0$ as $n \rightarrow \infty$. Each $\lambda_{n}$ is a finite pole, so each spectral projection $P_{n}$ is in $F$. Let $S_{n}=P_{1}+\ldots+P_{n}$, then find a self-adjoint projection $Q_{n}$ satisfying $S_{n} Q_{n}=Q_{n}$ and $Q_{n} S_{n}=S_{n}$. Let $V_{n}=Q_{n}-Q_{n-1}$, and define $U=\sum \lambda_{k} V_{k} \cdot U$ is clearly in $\bar{F}$ and $Q=T-U$ is shown to be quasinilpotent.

4. THEOREM 1 UQ - QU is quasinilpotent.

PROOF. For any $S \in A$, let $\tilde{S}$ denote the left regular representation of $S$. Then by Lemma 6.6 in Smyth [3], we have that $Q_{n} A$ is an invariant subspace of $\tilde{Q}$. since $Q_{n}=Q_{n} Q_{n}$, we have $Q_{n} \in Q_{n} A$. Hence $\widetilde{Q}\left(Q_{n}\right) \in Q_{n} A$, say $\widetilde{Q}\left(Q_{n}\right)=Q_{n} S$ for some $S \varepsilon A$. That is, $Q Q_{n}=Q_{n} S$. Now let $v \varepsilon$ range $Q_{n}$, say $v=Q_{n} \times$. Then $Q v=Q Q_{n} \times=Q_{n} S \times$ belongs to range $Q_{n}$. Hence we see that range $Q_{n}$ is an invariant subspace of $Q$. It follows that $Q$ has an operator matrix representation of the form 


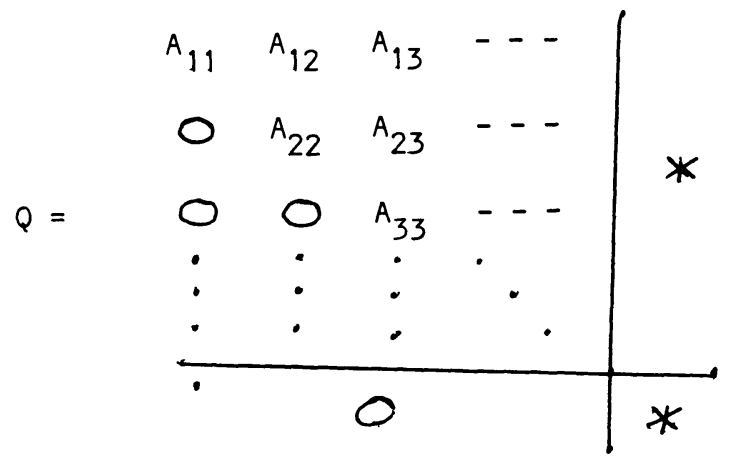

where $A_{i j}=V_{i} Q V_{j}$. With respect to this blocking, we have
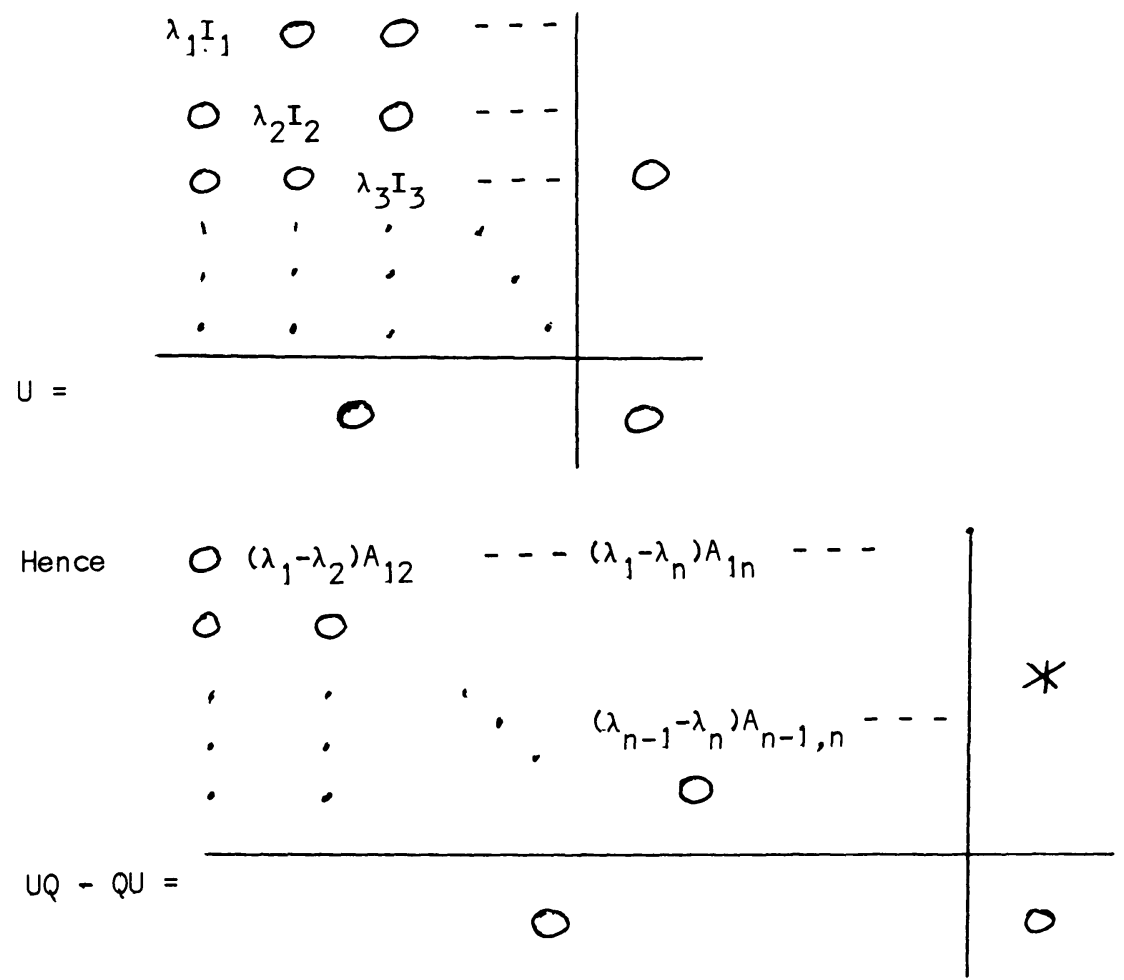
Now let $P$ be the orthogonal projection onto $\bigcup_{n}$ range $Q_{n}$, and let $A_{n}=\left(P-Q_{n}\right)(U Q-Q U)\left(P-Q_{n}\right)$. It is easy to see that ||$A_{n}|| \leq\left|\lambda_{n}\right| \| Q-$ diag. $Q|| \rightarrow 0$ as $n \rightarrow \infty$. Hence $\cup Q-Q U-A_{n}$ converges in the uniform norm to $U Q-Q U$ as $n \rightarrow \infty$. But $U Q-Q U-A_{n}$ has the form

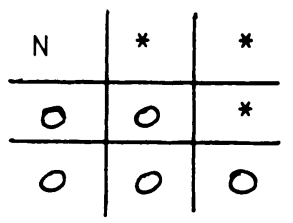

where $N$ is nilpotent. It follows that $U Q-Q U-A_{n}$ has no non-zero eigenvalues. Thm. 3.1, P. 14 of [2] can now be easily modified to show that UQ - QU has no non-zero eigenvalues. Since $U Q-Q U$ belongs to $\bar{F}$, this means $\sigma(U Q-Q U) \subseteq\{0\}$, i.e., UQ - QU is quasinilpotent.

\section{REFERENCES}

1. Chui, C. K., Smith, P. W., and Ward, J. D., A note on Riesz operators, Proc. Amer. Math. Soc., 60, (1976), 92-94.

2. Gohberg, I. C., and Krein, M. G., Introduction to the theory of linear nonselfadjoint operators, "Nauka," Moscow, 1965; English transl., Transl. Math Monographs, vol. 18, Amer. Math. Soc., Providence, R. I. 1969.

3. Smyth, M. R. F., Riesz theory in Banach algebras, Math Z., 145, (1975), $145-155$.

4. West, T. T., The decomposition of Riesz operators, Proc. London Math. Soc., III, Ser. 16, (1966), 737-752.

AMS (MOS) Subject Classification numbers $47 \mathrm{~B} 05,47 \mathrm{C} 10$

KEY WORDS AND PHRASES. $C^{*}$ algebra, quasinilpotent operators, Riesz elements. 


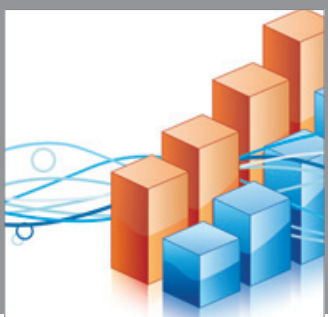

Advances in

Operations Research

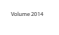

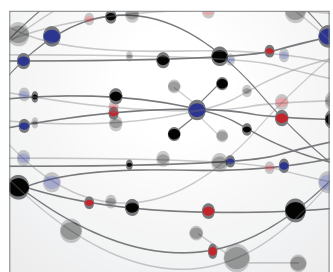

\section{The Scientific} World Journal
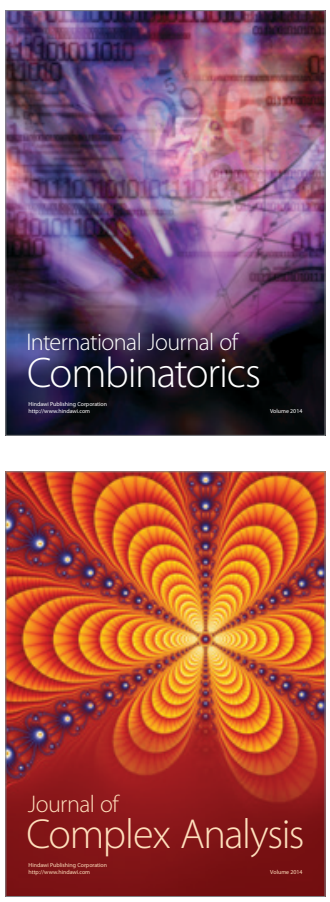

International Journal of

Mathematics and

Mathematical

Sciences
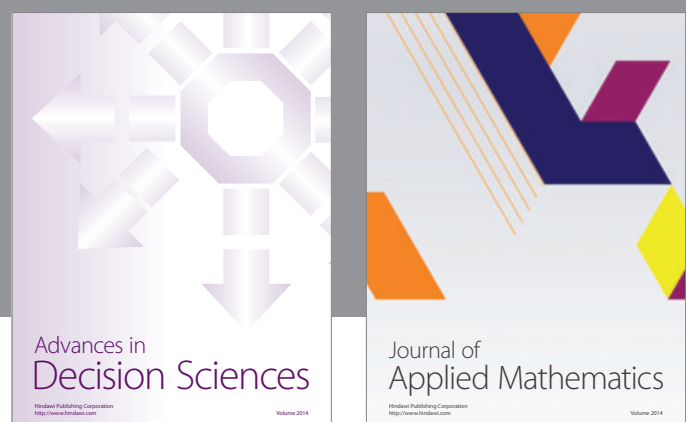

Journal of

Applied Mathematics
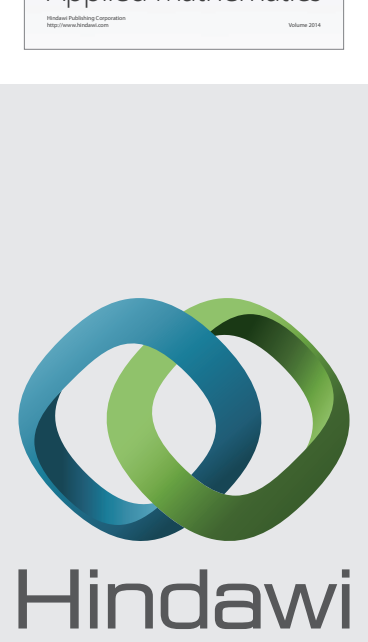

Submit your manuscripts at http://www.hindawi.com
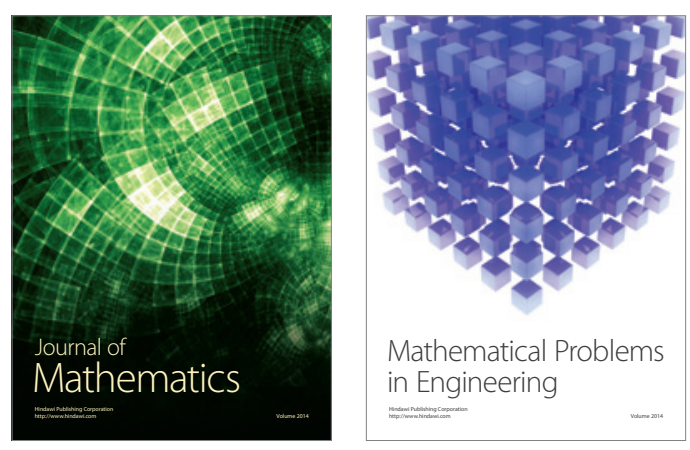

Mathematical Problems in Engineering
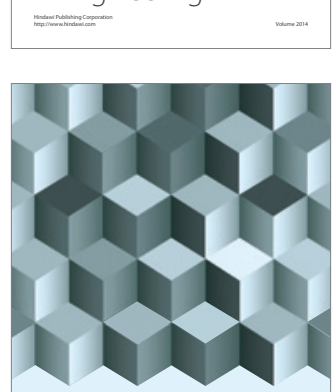

Journal of

Function Spaces
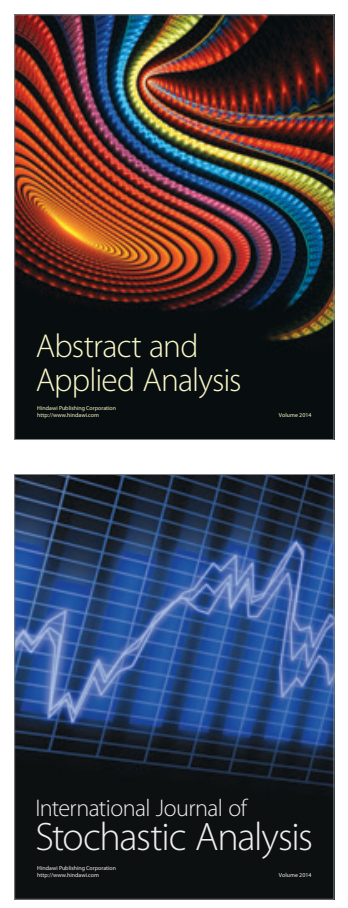

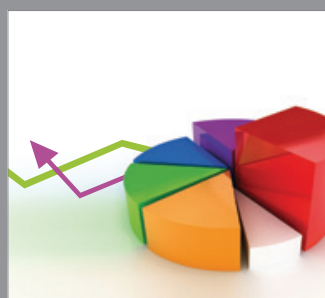

ournal of

Probability and Statistics

Promensencen
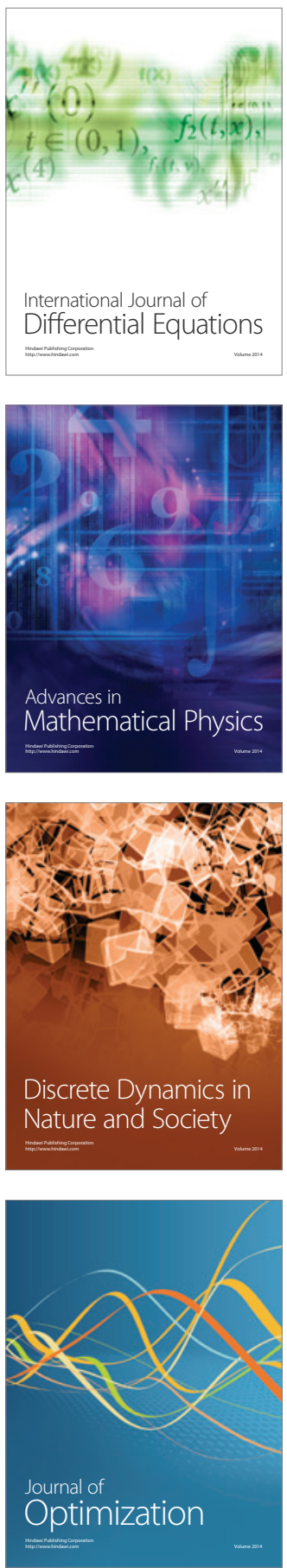